\title{
Über Wasserstoff- und Hydroxylionen- Gleichgewicht in Lösungen.
}

\author{
II. Mitteilung \\ von \\ W. Löfflep und K. Spipo.
}

(19. VIII. 19.)

Unsere Anschauungen über die Reaktionsverhältnisse in Zellen und tierischen Flüssigkeiten haben in den letzten Jahren wesentlich nach zwei Richtungen Förderung erfahren: einerseits ist es mit Hilfe der elektrometrischen Methode und der sich anschliessenden Indikatoren-Methode möglich gewesen, die Konzentration an H-Ionen mit einer Genauigkeit zu messen, welche die der meisten Methoden der analytischen Chemie weitaus überragt. Um die Ausarbeitung und Verwendung dieser Methoden haben sich besonders einerseits Sörensen und anderseits Michaelis und Rona und deren Mitarbeiter verdient gemacht. Als zweiter wesentlicher Fortschritt ist zu bezeichnen, dass L.J. Henderson ${ }^{\mathrm{j}}$ ) darauf aufmerksam gemacht hat, wie wichtig jene in allen tierischen Flüssigkeiten vorkommenden Stoffe sind, welche dafür sorgen, dass die Konzentration an H-Ionen meist dieselbe und einheitliche ist. Gerade Henderson. war es, der das speziell für die Carbonate und Phosphate gezeigt und damit erklärt hat, wie die Umwelt gerade in ihren Reaktionsverhältnissen geeignet ist, das Leben der Pflanzen und Tiere aufrecht zu erhalten. Es hat sich dementsprechend auch ergeben, dass die Konzentration an H-Ionen (im Folgenden immer ausgedrückt durch ihren negativen Logarithmus $p_{H}$ ) für die meisten tierischen Flüssigkeiten einè ganz bestimmte ist; z. B. ergab sich für das Blut die Konzentration $p_{H}=7,38$ bei $38^{\circ}$, die Reaktion der Organe ergab sich als ziemlich genau, neutral und nicht so alkalisch wie die des Blutes. Die Reaktion der Verdauungssäfte ist: für den Speichel

1) Das Gleichgewicht zwischen Basen und Säuren, Asher-Spiro's Ergebnisse der Physiol., Bd. 8, 254 (1909) und Monographie über die Eignung der Unwelt. Wiesbaden J.F. Bergmann, 1914. 
fast neutral, für den Magensaft deutlich sauer, für den Darmsaft ungefähr entsprechend einer verdünnten Bicarbonatlösung $p_{H} 7,7$, für die Milch ungefähr 7,0. Als spezifisch saure Flüssigkeit, die im Körper entsteht, kommt neben dem Magensaft noch der Harn in Betracht, dessen Reaktion nie die Alkalescenz des Blutes erreicht, und der höchstens so sauer werden kann, dass $\operatorname{sein} \mathrm{p}_{\mathrm{H}}=$ 5 wird. Während also der Harn Clärmer werden lann als das Blut, wird er nie H-ärmer.

Die Tatsache, dass der Organismus auf der einen Seite alkalischen Darmsaft, auf der andern Seite sauren Magensaft liefert, die Tatsache ferner, dass er einen Harn von in weiten Grenzen wechselndem Säuregehalt bereiten kann, zeigt, dass der Organismus imstande ist, die Konzentration an H-Ionen in ganz beliebiger Weise zu variieren. Es muss also dern Organismus ein doppeltes Vermögen gegeben sein, einerseits bestimmte Konzentrationen gleichmässig aufrecht $\mathrm{zu}$ erhalten, wofür ihm. die Regulatoren (Moderatoren, Puffer oder Dämpfer) dienen, andrerseits die Konzentration in sehr hohem Masse je nach dem Bedürfnis zu variieren.

Es ist eine allgemein feststehende Tatsache (und gerade hieraus erkennt man die Bedeutung der "Puffer" oder Moderatoren), dass die tierischen Zellen jenseits einer gewissen Säuerung nicht existieren können, und dass im allgemeinen ein erheblicher Alkaliverlust dem Körper schädlich ist. Das ist von um so grösserer Bedeutung, als im tierischen Organismus schon unter physiologischen Verhältnissen durch Oxydation fortdauernd Säuren entstehen, deren Menge unter pathologischen Bedingungen noch eine exzessive Steigerung erfahren kann. Man hat daher besonders die Vorgänge betrachtet, die zu einer Entsäuerung des Organismus führen, das ist normaler Weise einerseits die Atmung, die direkt vom H-Ionengehalt des Blutes abhängig ist, wo eine ausgesprochene Selbstregulation vorliegt, und andrerseits die Ausscheidung des Harns. Unter dem Gesichtspunkt der Regulation gewinnen aber auch intermediare chemische Prozesse im Organismus eine erhöhte Bedeutung, wie folgendes Beispiel zeigt:

Beim Diabetes mellitus treten bekanntlich (Minkowski) grosse Quantitäten $\beta$. Oxybuttersäure auf. Magnus-Levy hat eine Ausscheidung von über $342 \mathrm{gr}$ Säure in 3 lagen bei einem Kranken nachgewiesen. Dass die Bildung so grosser Mengen ron Säure für den Organismus eine grosse Gefahr bedeutet, 
ging nicht nur aus dem von den Kilinikern festgelegten Krankheitsbilde der Acjdosis hervor, sondern auch aus der experimentell festgelegten Tatsache, dass der Organismus zur Abwehr dieser Säuerung grosse Quantitäten Ammoniak zur Verfügung stellt. Neben diesem Neutralisationsvorgang greift der Organismus, um die starke Säuerung zu hemmen, aber auch synthetisch ein, wie sich aus folgendem ergibt: Durch die Untersuchungen von Neubauer, Dakin, Ernst Friedmann, Embden u. a. ist in den letzten Jahren nachgewiesen, dass die $\beta$-0xybuttersäure und zwar in der Leber zum überwiegenden Teil, wenn nicht ausschliesslich, durch Reduktion aus der Acetessigsäure entsteht, nicht wie man früher annahm, umgekehrt (ebenso Mandelsäure aus Phenylglyoxylsäure, Phenyl- $\beta$-oxypropionsáure aus Benzoylessigsäure). Der "Sinn“ dieser Reaktion (teleologisch gedacht) ergibt sich, wenn man aus den Dissoziationskonstanten beider Säuren (Acetessigsäure $1,5 \times 10^{-4}$ und $\beta$-Oxybuttersäure $3,86 \times 10^{-5}$ ), wie das zuerst Henderson und der eine von uns ${ }^{1}$ ) getan haben, berechnet, wieviel von den beiden Säuren im Harn als freie Säure und wieviel als Salz vorkommt. Dabei findet man unter der allerdings nur näherungsweise richtigen Voraussetzung, dass der Dissoziationsgrad der Na-Salze beider Säuren ceteris paribus ungefähr gleich ist, dass bei der in Betracht kommenden Reaktion des Harns $\left(p_{\mathrm{H}}=5\right.$ bis 6 ) die Acetessigsäure 3,3 bis 3,8 mal so stark dissoziiert ist als die $\beta$-0xybuttersäure, dass also durch die Reduktion der Ketosäure zur Oxysäure der Verlust an Alkali auf $1 / 3$ eingeschränkt wird. Es weist dies wiederum darauf hin, von welcher Bedeutung Aenderungen der Konzentration an $\mathrm{H}$ - und $\mathrm{OH}-$ Ionen für den Organismus und seine Funktionen sind.

Solche Änderungen der Konzentration an H- oder OH-Ionen in wässriger Lösung können auch durch Zusatz fremder Stoffe zustande kommen, nicht nur dadurch, was selbstverständlich ist, dass der zugesetzte Stoff selbst H- oder OH-Ionen enthält, sondern auch dadurch, dass er die Dissoziationsverhältnisse des Wassers und damit der gelösten Stoffe ändert. Das wird namentlich dort zu erkennen sein, wo die Reaktion der Lösung auf einer Reaktion des Wassers mit dem gelösten Stoffe beruht. Im allgemeinen ist man zwar berechtigt, in den tierischen Flüssigkeiten mit einer und derselben Dissoziationskonstanten des Wassers $\mathrm{k}_{\mathrm{W}}=10^{-14,14}$ bei $18^{\circ} \mathrm{zu}$ rechnen, da solche Konzentrationen an fremden Stoffen, die einen erheblichen Einfluss auf die $\mathrm{k}_{\mathrm{w}}$ des Wassers ausüben könnten (das sind meist mehr als molare Konzentrationen), in tierischen Flüssigkeiten nur selten sich finden dürften. Hingegen wissen wir, dass in Zellen, $d . h$. in heterogenen Systemen, an einzelnen Stellen Anhäufungen und erhebliche Konzentrationen vorkommen. Hierauf weist auch die Differenz in der $\mathrm{p}_{\mathrm{H}}$ zwischen Gewebe und Blut hin.

1) Henderson und K. Spiro, Bio. Z. 15, 105 (1908). 
Unsere im Reagensglas angestellten Versuche, über die im folgenden ganz kurz berichtet werden soll, können also infolge der hohen von uns angewandten Konzentration nicht ohne weiteres direkt auf die Verhältnisse im Organismus, speziell nicht auf dessen Flüssigkeiten, übertragen werden, aber einerseits wissen wir, wie schon hervorgehoben wurde, über die Konzentrationsverhältnisse, die Maxima der Speicherung, in einem mikroheterogenen System von der Art, wie es die Zelle ist, zu wenig, um darüber uberhaupt etwas sagen zu können; andererseits häufen sich die Erfahrungen, die zeigen, dass in einem so konzentrierten System, wie es das Zellprotoplasma ist, nicht die einfachen Verhältnisse verdünnter Lösungen gelten, wie sie uns die Elektrochemie speziell verdünnter Lösungen gelehrt hat ${ }^{1}$ ). Dafür sprechen auch die komplizierten Hydratations- und Solvatisierungsverhältnisse, die durch ein eingehendes Studium der Kolloïde immer mehr offenbar werden. Für die Biologie handelt es sich also im Folgenden einstweilen um Analogieversuche, die aber später einmal vielleicht die Brücke schlagen können von der physikalisch-chemischen zur rein chemischen Betrachtungsweise, und zwar zu Anschauungen, die sich auf chemischem Gebiet als überaus fruchtbar erwiesen haben.

In der anorganischen Chemie ist eine grosse Reihe von Reaktionen bekannt, bei denen ein Übergang einer nicht ionogenen Bindung in eine ionogene (z. B. Säure-) Bindung statt hat. Das genaue Studium gerade dieser Reaktionen hat $A$. Werner zu seiner Theorie geführt, die für die gesamte Lehre von der Valenz und damit für alle chemischen Anschauungen grundlegend geworden ist. Durch Anwendung dieser Erfahrungen und der Werner'schen Auffassungen auf die Vorgänge im Organismus können, wie wir vermuten, diese viel von ihrem bis dahin Rätselhaften verlieren. Das bisher noch nicht gelöste Problem der Salzsäurebildung z. B. liegt darin, dass man nicht weiss: woher die H-Ionen und woher gleichzeitig die Gl-Ionen kommen (nicht andere negative, z. B. $\mathrm{SO}_{4}{ }^{\prime \prime}$, sondern nur diese Cl-Ionen). Wir haben aber für das Auftreten der Gl-Ionen aus nicht-dissoziierten Verbindungen Analogien, und ebenso für das Auftreten von H-Ionen z. B. dort, wo durch Einlagerung von Molekeln eine an

1) Vgl, auch Michaelis und Rona, Bio. Z. 94, 239 (1919). 
sich schon vorhandene geringe Dissoziationstendenz erheblich gesteigert wird (s. unten).

$\mathrm{Zu}$ dieser anorganischen Reaktion haben wir nach Analogien bei organischen Umschau gehalten und zunächst diejenigen Stoffe herangezogen, die im Organismus hierfür in Betracht kommen könnten, die mehrwertigen Alkohole, sei es in Form der Kohlehydrate, sei es in Form des Glycerins (Fett).

Auf ein Verschwinden von OH-Ionen durch Zusatz von Glycerin hat der eine von uns schon immer im Unterricht hingewiesen, indem er zeigte, wie die Moor'sche Probe auf Zucker bei Gegenwart von Glycerin erheblich abgeschwächt wird. Eine. ähnliche Erscheinung lässt sich auch anschaulich machen, wenn man zu einer ganz verdünnten Baryt- oder Alkalilösung, die mit Phenolphthaleïn eben eine Rotfärbung gibt, Glycerin zusetzt, wodurch die Rotfärbung verschwindet. Umgekehrt braucht man mehr 0,1-n. Lauge, um in reinem Glycerin dieselbe intensive Rotfärbung gegen Phenolphthaleïn zu erzielen, wie in einer wässerigen Kontrollprobë. Dies steht in völliger Übereinstimmung mit Versuchen von Michaelis und Rona ${ }^{1}$ ), die in exakter Weise das Verschwinden von OH-Ionen auf Zusatz von Glycerin, mehrwertigen Kohlehydraten usw. elektrometrisch gemessen und daraus die Dissoziationskonstanten dieser Körper neu berechnet haben. Während aber Michaelis und Rona entsprechend ihren auf physikalisch-chemische Verhältnisse gerichteten Forschungen mit relativ geringen Zusätzen arbeiteten, haben wir umgekehrt, da wir von chemischen Gesichtspunkten ausgingen, mit hohen Konzentrationen (s. o.) gearbeitet. Umgekehrt haben Michaelis und Rona mit reinen Alkalilösungen gearbeitet, deren $\mathrm{p}_{\mathrm{H}}$ etwa zwischen 12,1 und 12,3 schwankte, also mit relativ starken Alkalilösungen, während wir in unsern Versuchen mit sehr viel verdünnteren, speziell mit sogenannten Pufferlösungen arbeiteten und uns mit Indikator-Messungen begnügten. Die auf diesem Weg erhaltenen Zahlen sind daher mit der dieser Methode eigenen Unsicherheit behaftet. Es kam uns aber zunächst nur darauf an (da es sich nur um Analogieversuche mit physiologisch-chemischen Verhältnissen handelt), das qualitative Verhalten festzustellen. Wir mussten einstweilen mit Rücksicht auf äussere Umstände auf die

1) Bio. Z. 94, 232 (1919). 
Festlegung absoluter Zahlen verzichten und uns mit den zur Orientierung, ausreichenden Indikatorbestimmungen begnügen. Alle Indikatorenversuche unterliegen selbstverständlich dem Bedenken, dass sich bei ihnen etwas einschleicht, was dem von Sörensen studierten "Salzfehler" entspricht ${ }^{1}$ ). Wenn der Salzfehler z. B. beim Phenolphthaleïn bei 3,5\% Salzgehalt -0,21 und bei $2 \%-0,16$ entspricht, so bedeutet das so viel, als dass unter dem Einfluss des Salzes das Phenolphthaleïn sich aus der lactoïden in die chinoïde Form umgewandelt hat. So sehr das die Feststellung absoluter Zahlen für die H-Konzentration durch Indikatoren erschwert, so ist doch die Salzwirkung als solche sehr interessant, denn sie zeigt uns, dass durch Salzzusatz manche Stoffe ihren Zustand ändern, d. h. sich so verhalten, als ọ sie sich in einem Milieu befänden mit einer andern Wasserstoffionenkonzentration als die elektrometrische Bestimmung angibt (s. u. S. 549). Die als Indikatoren benutzten Farbstoffe zeigen diese Änderung dadurch, dass sie Farbstoffe sind, nur besonders deutlich; aber es kann das, was bei ihnen wahrnehmbar ist, genau ebenso bei andern Körpern sich zeigen, die ebenfalls wie die Indikatoren schwache Säuren oder schwache Basen sind.

Wir haben z. B. in Versuchen, die an anderer Stelle ausführlicher veröffentlicht werden sollen, gefunden, dass der überlebende Dünndarm sehr empfindlich ist gegen Herabsetzung der Salzkonzentration in der umgebenden Flüssigkeit, und dass diese Wirkung reversibel ist.

Von den zur Herstellung von Pufferlösungen von Sörensen verwendeten Körpern haben wir zunächst seiner physiologischen Bedeutung wegen das Glykokoll herangezogen, dessen Säurekonstante nach Michaelis und Rona 1,2 $\times 10^{-10}$ und dessen Basenkonstante $1,93 \times 10^{-12}$ ist und dessen Lösung dementsprechend bei Geǵenwart von Phenolphthaleïn auf Zusatz eines Tropfens 0,1-n. NaOH rot wird. Bei Gegenwart von Glycerin (wir verwendeten entweder nach der Pharmac. Helv. gereinigtes Glycerin, oder ein Präparat von Kahlbaum) erhielten wir folgende Zahlen :

1) Vgh. Michaelis und Rona, Bio 7. 23, 61 (1909). Sörensen und Palitzseh, Bio. Z. 24, 387 (1910). 


\begin{tabular}{|c|c|c|c|}
\hline Glykokoll ca. $10 \%$ & $\mathrm{H}_{2} \mathrm{O}$ & Glycerin & $\begin{array}{c}\text { Titrationszahlen: } \\
0,1-\mathrm{n} . \mathrm{NaOH}\end{array}$ \\
\hline $1,0 \mathrm{~cm}^{3}$ & $4,0 \mathrm{~cm}^{3}$ & $0 \mathrm{~cm}^{3}$ & $1,0 \mathrm{~cm}^{3}$ \\
\hline 1,0 & 3.0 n & 1,0 & 2,0 \\
\hline 1,0 & 2,0 & $2,0 \quad$ & $2,6 n$ \\
\hline 1,0 & 1,0 & $3,0 \%$ & 3,4 \\
\hline $1,0 \quad$ & $0 \quad n$ & $4,0 \eta$ & $4,5 \quad$ \\
\hline
\end{tabular}

\begin{tabular}{|c|c|c|c|c|c|}
\hline $\begin{array}{c}\text { Glykokoll } \\
13 \% \\
\end{array}$ & $\mathrm{H}_{2} \mathrm{O}$ & Glycerin & $0,1-n . \mathrm{NaOH}$ & \multicolumn{2}{|c|}{$\begin{array}{c}\text { Dieselbe Reihe mit } \\
0,1-\mathrm{n} \text {. Glykokoll } \\
0,1-\mathrm{n} \text {. NaOH }\end{array}$} \\
\hline 0 & 4,0 & 8,0 & 0,25 & 0,03 & $\mathrm{~cm}^{3}$ \\
\hline 0,2 & 3,8 & 8,0 & 0,40 & 0,05 & $n$ \\
\hline 0,4 & 3,6 & 8,0 & 1,55 & 0,08 & $n$ \\
\hline 0,6 & 3,4 & 8,0 & 2,20 & $0,1.1$ & $n$ \\
\hline 0,8 & 3,2 & 8,0 & 2,80 & 0,14 & " \\
\hline 1,0 & 3,0 & 8,0 & $3,4 \check{5}$ & 0,16 & $"$ \\
\hline 1,2 & 2,8 & 8,0 & 4,10 & 0,18 & $"$ \\
\hline 1,4 & 2,6 & 8,0 & 4,65 & 0,20 & $n$ \\
\hline 1,6 & 2,4 & 8,0 & 5,10 & 0,22 & $n$ \\
\hline 1,8 & 2,2 & 8,0 & 5,75 & 0,24 & $\pi$ \\
\hline 2,0 & 2,0 & 8,0 & 6,40 & 0,27 & 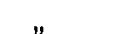 \\
\hline
\end{tabular}

Wir haben uns überzeugt, dass dieselbe Verschiebung beim Glykokoll auch nachweisbar ist mit anderen Indikatoren. Man kann sich davon sehr einfach überzeugen, indem man eine gesättigte Glykokollösung, ca. 3-n., mit Nitrophenol und Methylrot versetzt. Man sieht bei beiden Indikatoren, dass die glycerinhaltige Probe sauer ist, im Gegensatz zur alkalischen wässrigen;

$1 \mathrm{~cm}^{3} 3-\mathrm{n}$. Glykokoll $+3 \mathrm{~cm}^{3}$ Glyzerin $+5 \mathrm{Tr}$. Indikator'; Kontrollversuch mit $3 \mathrm{~cm}^{3} \quad \mathrm{H}_{2} \mathrm{O}$ :

\begin{tabular}{|c|c|c|}
\hline & $\mathrm{H}_{2} \mathrm{O}$ & Glycerin \\
\hline Methylrot & $\begin{array}{c}\text { alkalisch } \\
\text { gelb }\end{array}$ & $\begin{array}{l}\text { sauer } \\
\text { rot }\end{array}$ \\
\hline Nitrophenol & $\begin{array}{l}\text { alkalisch } \\
\text { gelb }\end{array}$ & $\begin{array}{l}\text { sauer } \\
\text { farblos }\end{array}$ \\
\hline Neutralrot & $\begin{array}{c}\text { sauer } \\
1 \mathrm{Tr} .0,1-n . \mathrm{NauH}\end{array}$ & $\begin{array}{c}\text { sauer } \\
2 \text { Tr. } 0,1-\mathrm{n} . \mathrm{NaOH}\end{array}$ \\
\hline
\end{tabular}


und bei Gegenwart von Neutralrot braucht man zum Umschlag nach der alkalischen Seite mehr Lauge in Gegenwart von Glycerin als in wässriger Lösung.

Wir haben dann weiter gesehen, dass auch beim Versetzen von Glykokoll mit Alkali sich mit Hilfe von Phenolphthaleïn zeigen liess, dass die Mischung von Glykokoll und Glycerin eine andere H-Reaktion hatte als eine solche in entsprechender wässeriger Lösung. Dies geht aus folgendem Versuchsprotokoll hervor:

$0,1-n$. Glykokolösung mit Phenolphthaleïn versetzt und Alkali $p_{H}=8,575$. Um dieselbe Färbung zu erzielen, waren bei steigenden Mengen Glycerin Alkalimengen nötig, die folgendem $\mathrm{p}_{\mathrm{H}}$ entsprachen:

\begin{tabular}{|c|c|c|c|}
\hline Glykokoll & $\mathrm{H}_{\mathbf{2}} \mathrm{O}$ & Glycerin & $\mathrm{p}_{\mathbf{U}}$ \\
\hline 1,0 & 3,0 & 1,0 & 9,016 \\
1,0 & 2,0 & 2,0 & 9,14 \\
1,0 & 1,0 & 3,0 & 9,36 \\
1,0 & 0 & 4,0 & 9,50 \\
\hline
\end{tabular}

Wir bereiteten uns eine Glykokoll-Natriumlösung, deren $\mathrm{p}_{\mathbf{H}}=8,57$ war, und setzten $z$ der oben genannten Mischung Glykokoll $+\mathrm{H}_{2} \mathrm{O}+$ Glycerin so viel Alkali zu, um eine Färbung zu erhalten, die der $p_{\mathrm{H}}=8,57$ entsprach. Da wir den Gehalt an Glykokoll und 0,1-n. NaOH kannten, ergab sich ohne weiteres aus den Sïrensen'schen Tabellen, welchem $p_{H}$ die Flüssigkeit ohne Gegenwart von Glycerin entsprochen hätte (letzte Spalte).

Auf diesem Wege liess sich zeigen, wie stark durch das Glycerin der H-Gehait verschoben wird, d. h. dadurch, dass man Glycerin zusetzte, konnte man eine Verschiebung bis um eine Zehner-Potenz erreichen. Kurz ausgedriuckt: Zu einer GlykokollGlycerinlösung muss man, um eine bestimmte $p_{H} z u$ erzielen, mehr NaOH zusetzen als zu einer rein wässrigen Lösung.

Es lag nahe, zu versuchen, ob man auf diesem Wege zu. einer quantitativen Titration des Glykokolls käme. Das gelingt aber nicht, auch wenn man grosse Quantitäten Glycerin anwendet; wohl aber gelingt es sehr leicht mit einem andern Alkohol, nämlich dem gewöhnlichen Aethylalkohol. Vorländer ${ }^{1}$ ) hat schon vor Jahren auf die Möglichkeit, Glykokoll in alkoholischer Lösung zu titrieren, hingewiesen. Er hat diese Erscheinung durch Bildung

1) A. 341,78 (1905). 
„innerer Addukte" erklärt, welche zum Teil den Charakter der quartären Ammoniumsalze haben, und im Gegensatz zu den gewöhnlichen Addukten aus Aminen und Säuren nicht leicht durch wässerige Alkalilauge zerlegt werden können ${ }^{1}$ ).

"Das innere Addukt des Glykokolls verbält sich also ähnlich wie die Halogen-Alkylate der tertiären Amine und nicht wie die einfachen Säureverbindungen der Amine, ein Unterschied, der namentlich bei den aromatischen Aminen sehr deutlich ist. Die Verbindung von Atom- und Molekül-Jon wird also nicht durch wässriges Alkali, wohl aber durch alkoholisches leicht gesprengt".

Mit diesen Erfahrungen Vorländers, dem verschiedenen Verhalten des Glykokolls in wässriger resp. alkoholischer Lösung, stimmen auch solche Emil Fischers ${ }^{2}$ ) überein, der bei der Darstellung des Säurechlorids $\mathrm{CH}_{2}\left(\mathrm{NH}_{2} \cdot \mathrm{HCl}\right)$-COCl einen Unterschied zwischen dem aus Wasser krystallisierten und dem mit Alkohol gefällten Glykokoll fand, und der schon mit aller Vorsicht die Möglichkeit zweier verschiedener Formen des Glykokolls erörterte. Durch Wirkung von Alkohol wird also der Säurecharakter und damit auch die chemische Reaktionsfähigkeit des Glykokolls geändert.

Man könnte zunächst geneigt sein. für das Glykokoll dieselben zwei Modifikationen in Betracht zu ziehen, die Hantzsch ${ }^{3}$ ) unter Annahme einer Betätigung der Werner'schen Nebenvalenzbindungen für die organischen Säuren vorgeschlagen hat: $\left.\mathrm{RC} \cdot \mathrm{O}_{2}\right\} \mathrm{H}$ als echte Säure und R.CO.OH als Pseudosäure. Dem widerspricht aber, dass in diesem Fall die stärkere echte Säure aus der schwächeren durch Alkohol entsteht, während Hantzsch gezeigt hat, dass die echten Carbonsäuren durch Alkohol in die Pseudosäuren übergehen.

- Bei den Aminosäuren haben wir gerade wegen der Aminogruppe besondere Verhältnisse: Früher nahm man schon oft innere Salzbildung an, an die aber z. B. Winkelblech ${ }^{4}$ ) "nicht glaubt“. G. Bruni und Fornara ${ }^{5}$ ), besonders aber $H$. Ley ${ }^{6}$ ) haben für die Kupfersalze der Aminosäuren, speziell des Glykokolls, nachgewiesen, dass es sich um innere Metallkomplexsalze handelt, bei

1) B. 52, 311 (1919).

4) Z. EI. Ch. 10, 293 (1904).

6) Z. El. Ch. 10, 954 (1904).
2) B. $38,2917(1905)$ 3) B. 50, 1422 (1917). 5) R. A. L. [5] 13, II, $26(1904)$. 
denen das Metall durch Nebenvalenz mit der Aminogruppe verbunden ist:<smiles>O=C1CCCCC1</smiles>

Neuerdings ist für die innern Komplexsalze die Bindung des Metalls an zwei verschiedene Atomgruppen bewiesen durch P. Karrer, C. Nägeli und H. Weidmann ${ }^{\mathbf{1}}$. Da nun die elektrolytische Dissoziation des Glykokolls sehr gering und sein Säure-Charakter eben nachweisbar ist, so ist wohl auch das Glykokoll in konzentrierter wässriger Lösung den inneren Komplexsalzen vergleichbar, d. h. eine Nebenvalenzbindung des $\mathrm{H}$ an die $\mathrm{NH}_{2}$-Gruppe anzunehmen, wodurch sich die Nichtdissoziationsfähigkeit ohne weiteres erklären würde. Bei Gegenwart von viel Wasser aber, ebenso durch Glycerin, am besten aber durch Alkohol wird die Nebenvalenz der Aminogruppen durch diese gebunden, sodass der saure Charakter des Glykokolls offenbar wird; das bisher durch Nebenvalenz in der ersten Sphäre festgehaltene H-Atom wird nunmehr ionogen in der zweiten Sphäre gebunden. Diese Annahme steht auch mit der Werner'schen Auffassung der Konstitution aller Säuren, z. B. dér Salzsäure, in vollkommener Harmonie: $\mathrm{HCl}+\mathrm{H}^{\cdot}+\mathrm{OH}^{\prime}=\mathrm{ClH} \cdot \mathrm{OH}^{\prime}+\mathrm{H}^{\cdot}$

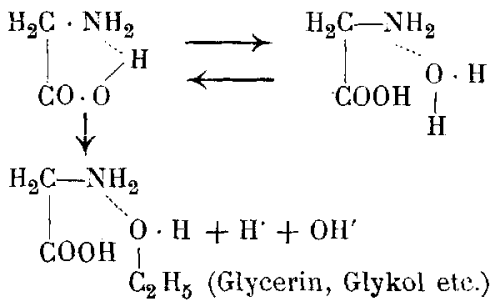

$$
\begin{aligned}
& \longrightarrow
\end{aligned}
$$

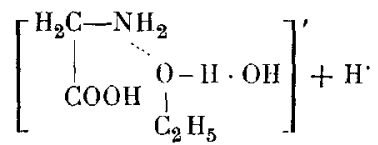

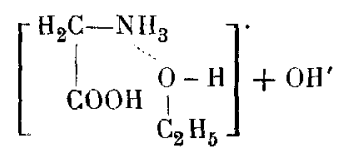

Man kann für diese Auffassung vielleicht noch folgende Erfahrung geltend machen: Wenn es sich bei der Einwirkung des Alkohols um die Auflösung eines inneren Komplexsalzes, d. h. das Ver-

1) Helv, 2, 242 (1919). 
schwinden der Nebenvalenzbindung an der Aminogruppe handelt, dann wäre zu vermuten, dass durch Alkohol nicht nur der saure, sondern auch der alkalische Charakter des Glykokolls mehr hervortreten müsste. Das ist in der Tat der Fall: wie gegen Phenolphthaleïn eben alkalische Alkohol- (resp. Glycerin-) Lösungen, mit ebensolchen Glykokollösungen zusammengebracht, nunmehr gegen Phenolphthaleïn sauer erscheinen, so werden gegen Methylorange eben saure Alkohol- (bezw. Glycerin-) Lösungen mit ebensolchen Glykokollösungen nunmehr wieder alkalisch. Auch bei der Titration gegen Methylorange liess sich das zeigen. Ähnlich dem Alkohol, nur mit geringerer Intensität, wirkt auch das Glycerin, die Wirkung wird aber durch vorhandenes Wasser gehemmt, wie uns besondere Versuche gezeigt haben.

Nichdem wir uns nun so überzeugt hatten, dass die alkalische Pufferlösung, die man aus Glykokoll herstellen kann, durch Zusatz erheblicher Glycerinmengen nach der sauren Seite hin verschoben werden kann, haben wir auch die andern alkalischen Pufferlösungen herangezogen. Ganz übereinstimmend ergab sich für sie alle, wie aus den folgenden Versuchsprotokollen hervorgeht, dass sie durch Glycerinzusatz ebenfalls nach der sauren Seite hin verschoben werden.

$1 \mathrm{~cm}^{3}$ Boratlösung titriert gegen Phenolphthaleïn auf Entfärbung.

\begin{tabular}{|r|r|r|}
\hline$p_{\mathrm{H}}$ & \multicolumn{3}{|c|}{ Dazu sind nötig: } \\
\hline \hline a) Glukose 20\% oder b) Glycerin
\end{tabular}

\begin{tabular}{|c|c|c|c|c|}
\hline \multirow{2}{*}{$\mathrm{p}_{\mathrm{H}}$} & \multicolumn{2}{|c|}{ Gegen Neutralrot $=6,8$} & \multicolumn{2}{|c|}{ Gegen Nitrophenol $=5,0$} \\
\hline & a) Glukose & b) Glycerin & Glycerin & Glukose \\
\hline 9,24 & $0,75 \mathrm{~cm}^{3}$ & $0,10 \mathrm{~cm}^{3}$ & $0,60 \mathrm{~cm}^{3}$ & \\
\hline 9,09 & $0,52 \quad$ & 0,075, & 0,51 & feste \\
\hline 8,91 & $0,37 n$ & $0,05 \quad n$ & 0,46 n & Substanz \\
\hline 8,68 & $0,29 \quad$ & 0,03 & $0,40 \quad$ & \\
\hline 8,29 & $0,25 \quad$ & 1 Tropfen & 0,38 n & \\
\hline
\end{tabular}


Es wurden dazu verwandt Boratlösungen, alkal. Citratlösungen, alkal. Phosphat-, alkal. Bicarbonat-Lösungen. Bei allen zeigte sich, das sie bei Glycerinzusatz weniger alkalisch resp. relativ sauer wurden. Da die Boratlösungen sehr empfindlich sind und sich mit ihnen die Verschiebung durch Glycerin viel leichter demonstrieren liess, haben wir diese für das Borat schon bekannte und viel verwandte Reaktion nach zwei Richtungen hin noch erweitert. Einerseits, indem wir statt Glycerin Glukose verwandten und zwar um zu zeigen, dass prinzipiell die gleiche Verschiebung wie durch Glycerin auch durch Zucker möglich ist. Wir haben dann ferner probiert, ob statt Glycerin auch andere Alkohole gebraucht werden können und haben dabei die umgekehrte Reihenfolge gefunden wie beim Glykokoll, indem nämlich das Glycerin wirksamer ist als das Glykol und dieses wieder wirksamer als Aethylalkohol. Aus den Versuchen geht weiter hervor - namentlich zeigen das die Versuche mit Glykol sehr deutlich - dass es sich um eine Massenwirkungsreaktion handelt, bei der man umso weniger von dem Alkohol braucht, je weniger Wasser vorhanden ist, dass es sich also gewissermassen um eine Verdrängungsreaktion des Wassers durch den Alkohol handelt. Wenn auch der Aethylalkohol nicht imstande ist, quantitativ in wässriger Lösung das eine H-Atom der Borsäure zu dissoziieren, so vermag er doch aus dem Hydrat der Säure Wasser zu verdrängen und damit die zur Erzielung des Endeffektes nötige Menge Glycerin herabzusetzen. Man kommt also bei der Titration von Borsäure mit weniger Glycerin aus, wenn man in alkoholischer, statt in rein wässriger Lösung die Bestimmung ausführt.

Dank den Feststellungen A. Werners über Koordinationsverbindungen kann man sich aber viel präzisere Vorstellungen darüber machen. Es ist durch die Forschung der letzten Jahre nachgewiesen, zunächst durch Miolati ${ }^{1}$ ) und durch Rosenheim ${ }^{2}$ ), dass aus Phosphorsäure, Kieselsäure und Borsäure leicht Heteropolysäuren entstehen (entsprechend ihrer Leitfähigkeit, Neutralisierungszahl usw.) Die Erfahrungen, die an den MolybdänPhosphorsäuren (-Arsensäuren) usw. gemacht worden sind, lassen

1) J. pr. [2] 77, 439 (1908).

2) Z. an. Ch. 69, 247, 261 (1910). 
sich ohne weiteres auf Mannit-Borsäure, Glycerin-Borsäure usw. übertragen, sodass nach der Werner'schen Anschauung die Glycerinborsäure sich ableitet von dem Grenztypus $\mathrm{H}_{9}\left(\mathrm{BO}_{6}\right)$, wobei dann entsprechend der Borwolframsäure $\mathrm{H}_{9}\left(\mathrm{~B}\left(\mathrm{OW}_{2} \mathrm{O}_{8}\right)_{8}\right)$ eine ebenso gebaute Glycerinborsäure ${ }^{1}$ ) bzw. Glykolborsäure bezw. Glukoseborsäure entsteht. $\mathrm{Ob}$ man dabei nach der ursprünglichen Werner'schen Auffassung von der Koordinationszahl 6 ausgeht, oder wie $P$. Pfeiffer ${ }^{2}$ ) neuerdings wahrscheinlich gemacht hat, auf Grund der Krystallstruktur, von der Koordinationszahl 12, ist für die vorliegende Frage nur von secundärer Bedeutung, sondern das wesentliche ist, dass durch Werners Theorie der komplexen Säuren wir nicht nur eine grosse Reihe von Analogien, sondern auch direkt eine Vorstellung dafür bekommen, wie ohne Aenderung der valenzmässigen Beziehungen doch durch das Einschieben von durch Nebenvalenzen gebundenen Molekelgruppen die Dissoziatonskonstante sich erheblich ändert. Gerade für die mehrwertigen Alkohole ist die Fähigkeit, solche Koordinationsverbindungen zu bilden, in weitem Umfange schon nachgewiesen. Es sei nur erinnert an die grosse Anzahl von meistens im Züricher Institut speziell von $A$. $\left(\right.$ Grün $^{3}$ ) und seinen Schülern dargestellten Verbindungen des Glykols und besonders des Glycerins mit Erdalkalimetallsalzen, an die vielen seit langer Zeit bekannten Doppelsalze der Hexite usw. Das für die vorliegende Frage wesentliche ist, dass derartige Komplexe sich offenbar auch schon in wässriger Lösung bilden, wie das aus unsern Indikatorversuchen hervorgeht.

In dieser Hinsicht sei noch besonders auf folgende von Grün gefundene Tatsache verwiesen: Die Mannitverbindungen mit Baryumhydroxyd und Strontiumhydroxyd zeigen in wässriger Lösung ein viel höheres Drehungsvermögen als reine Mannit-

$\left.{ }^{1}\right)$ In dieser Weise sind wohl auch die ähnlichen und physiologisch besonders interessanten Untersuchungen von $M$. Siegfried und S. Howwjanz (H. 59, 376 (1909)) über die Bindung von Kọhlensäure durch Alkohole, Zucker, Oxysäuren und ebenso durch Aminosäuren zu erklären. Erhöhung der Löslichkeit der Borsäure ist vielfach beobachtet, z. B. durch Harnstoff, Aceton, Salze u. s. w. Wir fanden bei Gegenwart von $20 \%$ Glukose eine Löslichkeitsverminderung um $15 \%$ bei Zimmertemperatur.

2) Z. an. Ch. 105, 26 (1918).

3) M. 37, $409(1916)$; B. 41, 3465 (1908); B. 45, 3359 (1912). 
lösungen gleicher Konzentration. Bei der Lösung der Komplexe findet demnach kein vollständiger Zerfall derselben statt. Wie Baryumhydroxyd und Strontiumhydroxyd, so verstärkt auch Calciumhydroxyd die Drehung von Mannitlösungen. Bei optischaktiven Verbindungen können die Drehungserscheinungen als willkommene, weil einwandfreie Kontrolle der durch Indikatoren gefundenen Resultate dienen.

Werner hat auf der einen Seite gezeigt, dass elektronegative Atome wie z. B. das Chlor im Chromchlorid, durch Einschiebung von Wasser mobilisiert werden können: $\left[\mathrm{Cr}\left(\mathrm{OH}_{2}\right)_{6}\right] \mathrm{Cl}_{3} \rightarrow$ $\rightarrow\left[\mathrm{Cr}\left(\mathrm{OH}_{2}\right)_{5} \mathrm{Cl}\right] \mathrm{Cl}_{2}+1 \mathrm{H}_{2} \mathrm{O} \rightarrow\left[\mathrm{Cr}\left(\mathrm{OH}_{2}\right)_{4} \mathrm{Cl}_{2}\right] \mathrm{Cl}+2 \mathrm{H}_{2} \mathrm{O}$.

Auf der andern Seite können, wie an der Phosphorsäure, Kieselsäure und Borsäure gezeigt worden ist, ebenso Wasserstoffatome mobilisiert werden, d. h. auf Grund der Werner'schen Lehre ist es ohne weiteres verständlich, dass in wässerigen Lösungen auch durch Einwivkung neutraler Molekeln Verschiebungen des $H^{-}$und $O H$-Ionen-Gleichgewichtes stattinden. Dass dies auch für die im tierischen Körper vorkommenden Stoffe eine Rolle spielt, ist eben gezeigt worden.

Wir haben nun versucht, ob wir nicht auch eine Änderung im umgekehrten Sinne erzielen können, d. h. ob nicht auch Pufferlösungen mit saurer Reaktion unter dem Einfluss des Glycerins alkalisch werden. Wir sind dabei davon ausgegangen, dass alle Alkohole Ampholyte sind, was ja gerade für das Glycerin bei der Fettspaltung mit alkoholischem Natrium nach Kossel. besonders klar hervortritt.

Wir haben unsere Untersuchungen zunächst an der Citronensäure gemacht und sodann an der Milchsäure. Wir haben diese beiden Säuren herangezogen, weil der Säurecharakter in beiden wohl zum Teil durch die OH-Gruppe bedingt ist; ist doch die Dissoziationskonstante der Milchsäure $1,35 \times 10^{-4}$, dagegen diejenige der Propionsäure $1,3 \times 10^{-5}$. Es war also zu vermuten, dass, wenn gewisse Affinitäten zwischen Glycerin und $\mathrm{OH}-\mathrm{Ver}-$ bindungen bestehen und dieselben sich bei der Milchsäure geltend machen würden, unter dem Einfluss des Glycerins eine Verschiebung nach der alkalischen Seite hin stattfinden würde. Die Versuche haben das in der Tat bestätigt; es eribrigt sich wohl, hierüber ausführliche Protokolle mitzuteilen. 
Auch in den nach Michaelis und Rona hergestellten Regulatorenlösungen von Essigsäıre und Natriumacetat konnten wir innerhalb der Breite $p_{\mathrm{H}}$ 3-4 durch Zusatz von Glycerin eine Verschiebung nach dem Neutralitätspunkt wahrnehmen mittels Methylorange, doch sind diese Versuche vielleicht nicht so eindeutig wie diejenigen mit Citronensäure. Auch bei der Titration von Glykokoll gegen Methylorange wird in glyceriniger Lösung mehr Säure gebraucht als in wässriger (s. o.).

Von der Erwägung ausgehend, dass die Anlagerung chemischer Körper und die physikalisch-chemische Adsorption zwei in ihrem Wesen ähnliche Vorgänge sind, und dass die Werner'sche Koordinationstheorie gewissermassen die Brücke zwischen beiden schlägt und auch bei der Aufklärung der Kolloïde mitzuwirken berufen ist, haben wir, wie wir schon in unserer ersten Mitteilung ${ }^{1}$ ) angegeben haben, auch die Adsorption der Puffer-, lösungen durch Tierkohle studiert. Es ergab sich hier ebenfalls, dass nach dem Schütteln mit Tierkohle die Reaktion dem Neutralitätspunkt zugeführt wurde, d. h. z. B. also ein Abnehmen der sauren Reaktion. Wir konnten das weiterhin bei Lösungen von Milchsäure und milchsaurem Natrium (Indikatoren: Methylrot und Methylorange) und von Ammoniak und Ammoniumchlorid (Phenolphthaleïn) bestätigen; das Phänomen ist besonders deutlich, wenn sich die Reaktion in der Nähe des Umschlagepunktes hält.

Diese Beobachtung, dass durch Schütteln mit Tierkohle die Reaktion bestimmter Lösungen mehr dem Neutralitätspunkt zugeführt wird, steht in bester Uebereinstimmung mit den neuesten Beobachtungen von Michaelis und Ron ${ }^{2}$ ), die im Maiheft der biochemischen Zeitschrift dieses Jahres in sehr exakten Versuchen gezeigt haben, dass von allen Ionen die $\mathrm{H}$ - und $\mathrm{OH}-I o n e n$ weitaus am besten adsorbiert werden. Unter diese allgemeine Regelmässigkeit fällt also auch unsere Beobachtung, dass aus einem Gemisch von einer schwachen Säure und ihrem Salz resp. schwachen Base und ihrem Salz die Säure resp. Base stärker

1) Helv. 2, 417 (1919).

2) Handb. der Biochemie, Artikel Adsorption. 
adsorbiert wird als das Salz. Das ist für die Reaktionsverhältnisse im Tierkörper von Bedeutung, was hervorzuheben wir für wichtig halten, weil ja gerade durch die Kombination von schwacher Säure (Base) und Salz die Reaktionsverhältnisse im Tierkörper innegehalten resp. gedämpft werden (Puffer, Moderatoren).

Wenn man, wozu man theoretisch berechtigt ist (s. o.), den adsorbierenden Körper als ein Lösungsmittel ansieht, so sind alle diese Erfahrungen ohne weiteres in Analogie zu setzen mit den Beobachtungen über die Spaltung saurer Salze durch ein zugefügtes Lösungsmittel, worüber gerade aus jüngster Zeit Erfahrungen vorliegen ${ }^{1}$ ).

Wir brauchen die Theorie dieser Vorgänge hier nicht zu geben, da sie ja in aller Ausführlichkeit erst kürzlich von Dieckmann und Hardt ${ }^{2}$ ) mitgeteilt ist. Wir haben, gerade um die Analogie von Adsorption und Lösung besonders deutlich zu demonstrieren, die Adsorption durch Tierkohle an einem besonders charakteristischen Fall studiert: an den sauren Natriumsalzen der Fumar- und Maleïnsäure. Bei diesen beiden Säuren, die sich ja nur durch ihre sterische Konfiguration unterscheiden, liegen die beiden Dissoziationskonstanten $K_{1}$ und $K_{2}$ nach Landolt-Börnstein bei $9,4 \times 10^{-4}$ und $3,2 \times 10^{-5}$, bezw. bei $1,4 \times 10^{-2}$ und $2,6 \times 10^{-7}$, $\frac{K_{1}}{K_{2}}$ ist also für Fumarsäure $=29$, für Maleünsäure aber $=5385$.

Die Adsorbierbarkeit beider Säuren [0,29 gr gelöst in $\left.250 \mathrm{~cm}^{3}\right]$ durch Tierkohle erwies sich als gleich, nämlich $84 \%$ bei Zimmertemperatur. Es ist dies wichtig festzustellen, weil sie sich bei der Chemotaxis, wie $W$. Pfeffer zeigte, verschieden verhalten.

$10 \mathrm{~cm}^{3}$ der ursprünglichen Natriumhydrofumaratlösung entsprachen

$20 \mathrm{~cm}^{3} 0,1-n$. NaOH gegen Phenolphthaleïn

und gaben $0,141 \mathrm{gr} \mathrm{Na}_{2} \mathrm{SO}_{4}$

$10 \mathrm{~cm}^{3}$ der ursprünglichen Natriumhydromaleïnatlösung entsprachen

$20 \mathrm{~cm}^{3} 0,1-\mathrm{n}$. NaOH gegen Penolphthaleïn

und gaben $0,142 \mathrm{gr} \mathrm{Na}_{2} \mathrm{SO}_{4}$

Je $25 \mathrm{~cm}^{3}$ beider Lösungen wurden mit je $1,5 \mathrm{gr}^{2}$ Tierkohle geschüttelt. Vom Filtrat entsprachen je $10 \mathrm{~cm}^{3}$

1) H. Thomas und Sabalitschki, B. 50, 1227 (1917); 52, 567 (1919).

2) W. Dieckmann und A. Hardl, B. 52, 1134; Mc. Coy, Am. Soc. 30, 688 (1908). 
bei der Fumarsäure $7 \mathrm{~cm}^{3} 0,1-\mathrm{n}$. $\mathrm{NaOH}$ (Phenolphthaleïn) und $0,124 \mathrm{gr} \mathrm{Na}_{2} \mathrm{SO}_{4}$ " Naleïnsäure $14,0,1-\mathrm{n}: \mathrm{NaOH} " \# \quad 0,114 \mathrm{gr} \mathrm{Na}_{2} \mathrm{SO}_{4}$

d. h. das Verhältnis von einem Aequivalent II zu einem Aequivalent Na ist in der ursprünglichen Fumaratlösung $=2: 1,99=1: 1$

$" \quad$ Maleïnatlösung $=2: 2,0=1: 1$

im Filtrat der geschüttelten Fumaratlösung $=0,7: 1,75=1: 2,5$

" " " Maleïnatlösung $=1,4: 1,60=1: 1,14$

a. h. beide Flüssigkeiten waren, was auch. die Indikatorenprüfung bestätigte, nach dem Schütteln weniger sauer, der Säureverlust war aber bei der Fumarsäure mehr als doppelt so gross $(2,2)$ als bei der Maleïnsäure. Auch in zwei andern Versuchen ergab sich dasselbe Resultat: 2,2 resp. 2,1 mal so viel H verschwanden aus der Fumarsäurelösung.

Diese Beobachtungen zeigen, da die adsorbierte Menge eines Stoffes der vorhandenen Menge proportional ist, dass die wässrigen Lösungen der sauren Salze bei der Fumarsaäure mehr freie Säure enthalten, als bei der stereoisomeren Maleïnsäure.

Es ist wahrscheinlich, dass die Aenderung der H-IonenKonzentration, die, wie oben gezeigt wurde, durch Glycerin, mehrwertige Alkohole, Glukose u. s. w: erzielt wird, auch sonst durch Zusatz anderer Stoffe erreicht werden kann. Hierfür sprechen schon Erfahrungen, die z. B. an Ghromchloridlösung bei Zusatz von neutralen Chloriden gemacht worden sind ${ }^{1}$ ). Da die H-Ionen-Konzentration bei Salzzusatz umso mehr steigt, je grösser die Zahl der Wassermolekeln ist, die nach der HydrationsTheorie sich mit dem Salz vereinigen, so liegt auch hierfür die Anwendung der Werner'schen Anschauung nahe.

Aus diesen Beobachtungen ergibt sich deutlich auch ein Einfluss der Salze auf die Reaktion neutraler Körper, ein Einfluss, der freilich bisher bez. der im Tierkörper vorkommenden Stoffe trotz vielfacher Versuche nicht einwandfrei festgestellt werden konnte (s. aber oben "Salzfehler").

Wir möchten also das wesentliche Resultat unserer Versuche wie folgt zusammenfassen:

1) Baldwin, C. 1919, I. 805; Wilson und Kern, ebenda. 
1. Die einfachen neutral reagierenden Bausteine der tierischen Zelle: Glycerin, Kohlehydrate u s. w. sind im Sinn der A. Werner'schen Koordinationstheorie durch Bildung von Komplexsalzen imstande, Reaktionsverschiebungen hervorzurufen, speziell auch innerhalb der Breite, wie sie im tierischen Organismus vorkommen. Das lässt sich z. B. zeigen an dem einfachsten Baustein der Eiweisstoffe, dem Glykokoll.

2. Auch solche Lösungen, die gegen eine Änderung ihrer Alkalescenz sehr unempfindlich sind (Puffer), ändern beim Schütteln mit Tierkohle ihren Gehalt an $\mathrm{H}$ - und $\mathrm{OH}-I o n e n$ in der Art, dass sie dem Neutralitätspunkt näher gebracht werden.

3. In Konsequenz der vorgetragenen Anschauung, dass die Adsorption mit Tierkohle einem Lösungsvorgang gleichzusetzen ist, wird eine verschiedene Spaltung saurer Salze in wässeriger Lösung auch durch Schütteln mit Tierkohle gezeigt.

Frl. Mariz: Becker danken wir bestens für ihre freundliche Hilfe.

Basel, Laboratorium der medizinischen Universitätsklinik.

\section{Sur une nouvelle séparation de l'aluminium et $d u$ vanadium}

pat

P. Wenger et H. Vogelson.

(31. VIIJ. 19.)

$\mathrm{Au}$ cour's d'une étude complète du vanadium au point de vue analytique, nous avons eu l'occasion d'étudier Jes principaux dosages de cet élément ainsi que plusieurs de ses séparations, notamment d'avec le fer et l'aluminium.

Nous avons pu nous rendre compte qu'un grand nombre de ces méthodes ne. donnent pas des résultats satisfaisants ou présentent des difficultés techniques qui nécessitent une longue expérience pour arriver au but poursuivi. 